\title{
Performance Analysis of Cooperative Communication with Wireless Energy Harvesting Relays Over Nakagami-M Fading Channels
}

\author{
K. Pavani, V. Annapurna \\ Electronics and Communication Engineering, Jawaharlal \\ Nehru Technological University, Anantapur \\ Email:pranusha.lokesh@gmail.com
}

\begin{abstract}
In this paper, decode-to-forward cooperative system is considered with wireless energy harvesting relays. Relays are spatially randomly located and have the finite storage battery. Relays can harvest the energy from the RF signals, broadcasted by the destination. OSRL scheme is employed to improve the system performance. For characterising the performance of proposed scenario, exact closed form analytical expressions for the outage probability are derived and also derive the expression for the system throughput. Finally simulation results are presented to show that the system performance is increased by increasing the energy harvesting time.AC-DC conversion efficiency has also shows effect on the system performance.
\end{abstract}

Keywords-Perfect CSI, outage probability, Nakagami-m fading.

\section{INTRODUCTION}

Now a days need for the high data rate and capacity is increased due to daily life applications. Cooperative communication is the key technology which is used in current and future wireless networks. Relays can forward the source information to the destination to increase the coverage and save the transmit power.

Energy harvesting (EH) can play a main role to improve the system performance [2].In this paper we considered wireless energy harvesting to achieve the system performance. Sensors needs the DC power while remain active in the network [3].Traditional energy harvesting techniques are there like wind energy harvesting, hydal energy harvesting, tydal energy harvesting but we prefer RF energy harvesting because RF signals carry the information and power simultaneously [4].Power splitting (PS) protocol enables the receiver to perform the EH and ID separately [5,6].In this paper we consider the time- switching protocol (TS) enables the receiver to perform the two functions at the same time. Relaying protocols were proposed to enable the EH and ID at the relay node.

Two relaying protocols namely PSR and TSR were proposed in [7] to activate the EH and ID at the relay. Cognitive relay is considered for the energy harvesting in the presence of multiple transmitters and receivers [8].Relays assist the communication between transmitter and receiver. Transmitter and receiver cannot communicate due to deep fading then with the help of the relay they can communicate. Here relay decode the information which is sent by the transmitter and forward that information to the destination. Energy harvesting is the technology to extend the life of the battery. Here relay is the energy harvesting relay means it can extract the power from the RF signals which is broadcasted by the destination and used that extracted power to send information to the destination.

NOMA (non orthogonal multiple access) scheme is employed in [9] in which we have to consider the user locations to determine the performance. Near user selected as energy harvesting user to support the far user. Two relay selection schemes were proposed to select the best one to forward the source information to destination over Nakagami-m fading channel [10].Antenna selection scheme and generalized selection combining is employed in [11].Relay and source collect the energy in the downlink and worked in a cooperative manner in uplink [12].In [13] reduce the percentage of outage in presence of slow fading channel. We will consider the switching policy between energy harvesting and data relaying in [14].Relay selection scheme under spatially located random relays in [17]. 


\subsection{Motivation and contributions}

Comparing these works with the existing works Rayleigh fading was considered in $[7,10,11,12,13,14]$. Battery with infinite model adapted in [7-15].Selection of relay has not considered in [7-10].Static relay were adapted in [7-10,12-15].Nakagami fading channel is the hybrid channel. It can reduce to different types of channel by varying the shaping parameter $\mathrm{m}$. It can reduce to Gaussian channel if $m=\frac{1}{2}$ and reduce to Rayleigh channel if $m=1$.We can evaluate the performance of DF relaying SWIPT based cooperative system under the case, which based on availability of CSI. If perfect CSI is available OSRL scheme is employed.

- OSRL scheme: Here relays equipped with multiple antennas and adopt MRC scheme. Source has single antenna. Performance is increased by using MRC scheme.

The main contributions of this work are summarized as given below.

(1)We characterize the PDF and CDF of the SNR of the links S-R and R-D.

(2)We can derive the expressions for the outage probability..

In existing methods no works have studied OP of cooperative DF relay in the presence of Nakagami-m fading channels.

\subsection{Organization}

The rest of the paper can be divided as follows. In section II, OSRL scheme is considered. In OSRL scheme EH relay equipped with multiple antennas, MRC scheme is employed to improve the performance. In section III, numerical results are presented for OP .Finally in section IV, we can conclude with this paper.

\section{OSRL SCHEME}

In Fig.1 system model is considered. Disc is denoted by $\mathrm{C}$ with radius $R_{C}$. One single antenna source placed at the centre of the disc denoted by T. Multiple relays $R_{m}(\mathrm{~m}=1,2, \ldots . \mathrm{M})$ are randomly located within the disc. The location of relays are poisson point process with intensity $\emptyset$. One destination $\mathrm{D}$ placed at the distance $\mathrm{d}$ from the source. Due to deep fading source and destination cannot communicate .They can communicate with help of the relays. Here assume that size of all batteries is same. $N_{1}, N_{2}$ are the number of receiving antennas at the destination and relay $\left(N_{1} \geq 1\right)$ and $\left(N_{2} \geq 1\right)$. MRC scheme is employed at the relay and destination to improve the diversity gain. We can assume distance between source and all relays is same .This distance is negligible compared with the distance between source and destination. $d_{T R}$ is distance between source and best relay. Relays inside the disc are homogenious poisson point process with distribution $P_{r}(k)=$ $\frac{\lambda^{k}}{k !} e^{-\lambda}, \lambda=R_{C}^{2} \pi \varnothing \quad$ is the mean and $\varnothing$ is the intensity of PPP. $T_{C}$ is the total communication time.

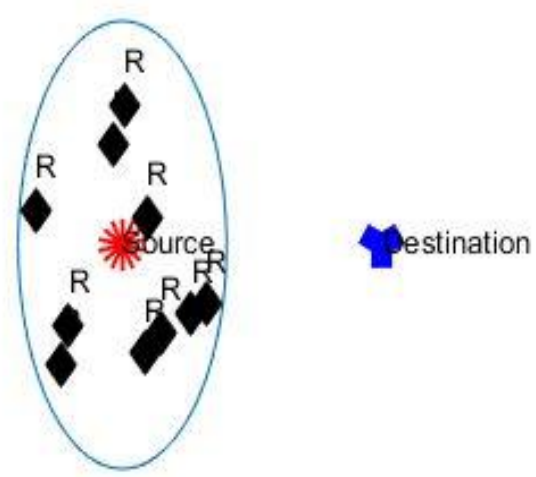

Fig.1. System Model

Whenever TS strategy is employed at the DF relay total communication time is divided in to three time slots. During the first time slot $(1-\mu) T_{C_{1}}$ (where $0<\mu<1$ ) relay forward the CSI of $\mathrm{T}--R_{m}$ channels to the source and source select the best relay to forward information based on CSI from relays. And intimate the selection results to relays and send the information to the best relay. During the second time slot $\mu T_{C_{1}}$ energy harvesting process takes place. Batteries with the size $B$ at the relays are charged by the signals, which are broadcasted by destination. AC to DC conversion circuit is there at the relays. Rectenna, which capture the RF signals from the destination and extract the DC voltage from the AC voltage. Relay used that extracted power to forward the message to the destination. Finally, remaining time slot $T_{C_{2}}$ $=T_{C}-T_{C_{1}}$ best relay forward the information to the destination. Let us assume that $\sigma=\frac{T_{C_{1}}}{T_{C_{2}}}$, and $h_{L}$ $, \boldsymbol{h}_{L},(\mathrm{~L} \in\{\mathrm{DR}, \mathrm{TR}, \mathrm{RD}\})$ are the average channel power gains and channel vectors of $\mathrm{D}-\mathrm{R}, \mathrm{T}-\mathrm{R}$ and $\mathrm{R}-\mathrm{D}$ links.

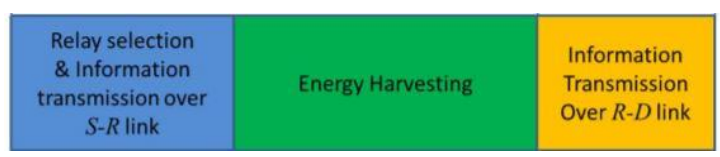

Fig.2. TS strategy

\subsection{Signal model}




\section{Available online at www.ijrat.org}

Under OSRL scheme we can select optimal source relay link. There are $\mathrm{M}$ number of relays randomly deployed in the disc C. So M-Independent links are there. Those links are independent and identically distributed. PDFs and CDFs of those channels are $f\left(g_{L}\right)=\left(\frac{m_{L}}{h_{L}}\right)^{N_{n} m_{L}} \frac{g_{K}^{N_{n} m_{L}-1}}{\Gamma\left(N_{n} m_{L}\right)} \exp \left(-\frac{m_{L} g_{L}}{h_{L}}\right)$

And

$$
\begin{aligned}
& F\left(g_{L}\right)= \\
& 1-\exp \left(-\frac{m_{L} g_{L}}{h_{L}}\right) \sum_{i=0}^{N_{n} m_{L}-1} \frac{1}{i !}\left(\frac{m_{L} g_{L}}{h_{L}}\right)^{i}
\end{aligned}
$$

Here $m_{L}$ is called the shaping factor of Nakagami channel over L link. $\Gamma($. $)$ is the gamma function. $N_{n}$ is the number of antennas at node $\mathrm{n}$ ( $\mathrm{n} \in\{R, D\}$ ). Among those links we can select the optimum link means we can select the best relay. The relay which has the maximum SNR can be selected as the best relay that is, $R=\max _{1 \leq m \leq M}\left\{\gamma_{T R_{m}}\right\}, \gamma_{T R_{m}}$ is the SNR of the link from $\mathrm{T}$ to $R_{m}$ channels. During the first time slot received signal at the relay $\boldsymbol{y}_{T R}=$ $\frac{h_{T R} x_{T} \sqrt{P_{T}}}{d_{T R}^{\alpha}}+n_{R}$. Here $x_{T}$ is the message from the source and $\alpha$ is the path loss exponent, $P_{T}$ is the transmitted power at the source. During the energy harvesting mode energy extracted by the relay is $E=\frac{\eta \mu\left\|\boldsymbol{h}_{D R}\right\|^{2} P_{D} C_{1}}{d^{\alpha}}$, where $\eta$ is $\mathrm{AC}$ to $\mathrm{DC}$ conversion efficiency at the relays, $P_{D}$ is the transmitted power at the destination. $n_{R}, n_{D}$ are the Additive White Gaussian Noises at the relay and destination nodes with mean 0 and variance $N_{0}$. Size of the battery shows impact on the system performance.Battery transmission power with battery size $B$ depends on two cases

$$
P_{R}= \begin{cases}P_{E}, & E<B \\ P_{B}, & E \geq B\end{cases}
$$

In this paper we assume that all the batteries have the same size. When size of battery is more then it can have the capability to store the more DC power which is extracted from the RF signals. If relay has the more transmitted power SNR at the destination is increases. If SNR at the destination is increases outage probability at destination is decreases and throughput increases. The probability that battery has more size compared to extracted energy

$$
\begin{aligned}
P_{E_{1}}^{O S R L}(B) & =\operatorname{Pr}\left[\frac{\mu \eta T_{1}\left\|h_{D R}\right\|^{2} P_{D}}{d^{\alpha}}<B\right] \\
& =1-\exp \left(-\beta_{D R} B\right) \sum_{k=0}^{N_{2} m_{D R}-1} \frac{\left(\beta_{D R} B\right)^{k}}{k !}
\end{aligned}
$$

The probability that battery has less size compared to compared to extracted energy

$$
\begin{array}{r}
P_{E_{2}}^{O S R L}(B)=\operatorname{Pr}\left[\frac{\mu \eta P_{D}\left\|h_{D R}\right\|^{2} T_{C_{1}}}{d^{\alpha}} \geq B\right] \\
=\exp \left(-\beta_{D R} B\right) \sum_{k=0}^{N_{2} m_{D R}-1} \frac{\left(\beta_{D R} B\right)^{k}}{k !},
\end{array}
$$

Where $\beta_{D R}$ is given in [1].

Finally after performing energy harvesting received signal at the destination can be calculated as

$$
y_{R D}=\frac{h_{R D} x_{R} \sqrt{P_{R}}}{d^{\alpha}}+n_{D}
$$

The data rate supported by the channel $\mathrm{S}$ to $R_{m}$ and $\mathrm{R}$ to $\mathrm{D}$ is $R_{T_{m}}=\frac{1}{2} \log \left(1+\gamma_{T R_{m}}\right)$ and $R_{R}=\frac{1}{2} \log \left(1+\gamma_{R D}\right) . \quad \gamma_{T R_{m}}, \gamma_{R D}$ are the SNRS at the relay and destination nodes.

\subsection{Outage probability}

Outage probability is defined as the probability that instantaneous SNR lesser than the target SNR. Under OSRL scheme assuming perfect CSI scenario outage probability is given as

$$
\begin{aligned}
P_{\text {out }}^{\text {OSRL }}\left(\chi_{T}, \chi_{R}\right)= & P_{E_{1}}^{O S R L}(B) O P_{1}\left(\chi_{T}, \chi_{R}\right) \\
& +P_{E_{2}}^{O S R L}(B) O P_{2}\left(\chi_{T}, \chi_{R}\right)
\end{aligned}
$$

Here $O P_{1}$ and $O P_{2}$ are explained in ref [1] and $P_{E_{1}}^{O S R L}$ and $P_{E_{2}}^{O S R L}$ are given from (4) and (5).

Table 1. Comparison table

\begin{tabular}{|l|c|c|}
\hline Technique & OP & $h_{R D}$ \\
\hline Existing & $10^{-3}$ & 22 \\
\hline proposed & $10^{-4}$ & 22 \\
\hline
\end{tabular}

Table 2. SD versus MRC

\begin{tabular}{|l|c|c|}
\hline Technique & OP & $h_{R D}$ \\
\hline SD & $10^{-2}$ & 10 \\
\hline MRC & $10^{-3}$ & 10 \\
\hline
\end{tabular}

\section{NUMERICAL RESULTS}

By using the Monte Carlo simulation we can evaluate the performance of OP. 


\section{Available online at www.ijrat.org}

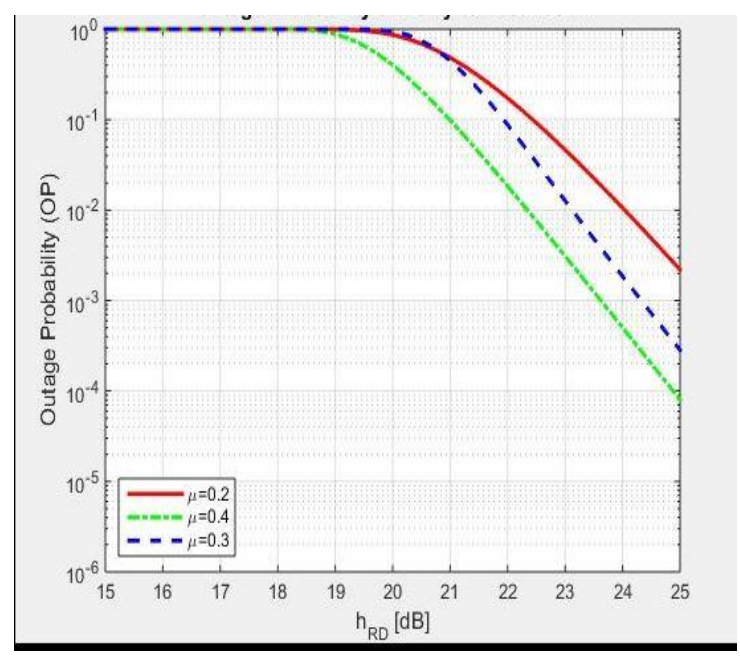

Fig.3. OP versus $h_{R D}$ for $N_{T}=4, N_{1}=4, h_{T R}=$ $10, T_{C_{1}}=450, P_{T}=45 \mathrm{~mW}$

As shown in figure 3,Op can decreased by increasing the energy harvesting time .Energy harvesting time increases by increasing the $\mu$.If energy harvesting time is large then there is possibility to extract the more power by the relay from the RF signals. If transmitted power at the relay increases SNR at the destination increases.

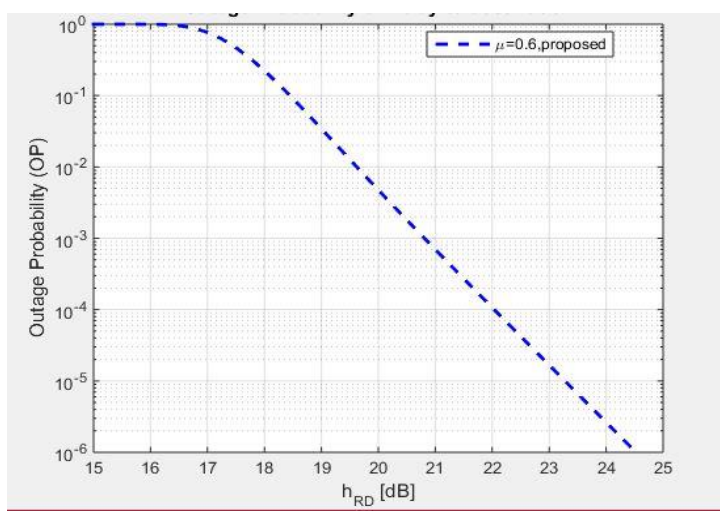

Fig.4. OP versus $h_{R D}$ for $N_{T}=4, N_{1}=4, P_{D}=$ $60 \mathrm{~W}, T_{C_{1}}=500$

From the figure 4 we can conclude that compared to existing work OP improved in our work.

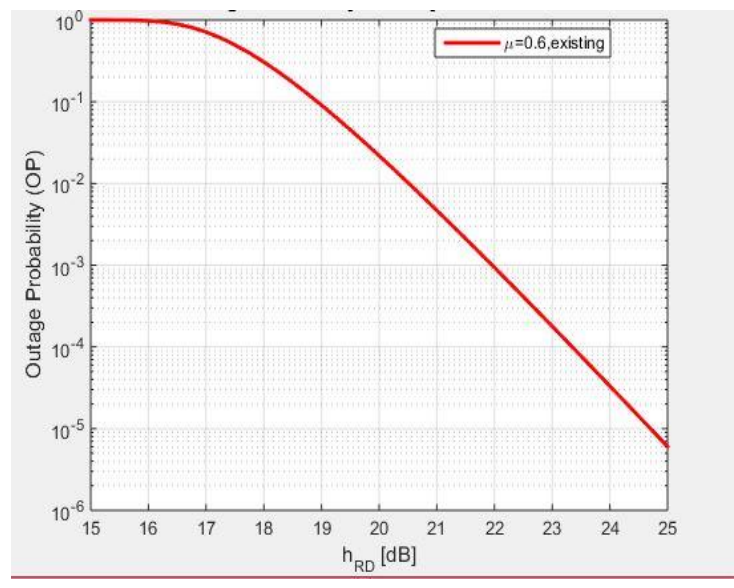

Fig.5. OP versus $h_{R D}$ for $N_{T}=4, T_{C_{1}}=500$

$$
\mathrm{Mw}, h_{T R}=10
$$

Fig.5 represents the OP versus $h_{R D}$ for given energy harvesting time.Relay selection has not been considered in existing work.

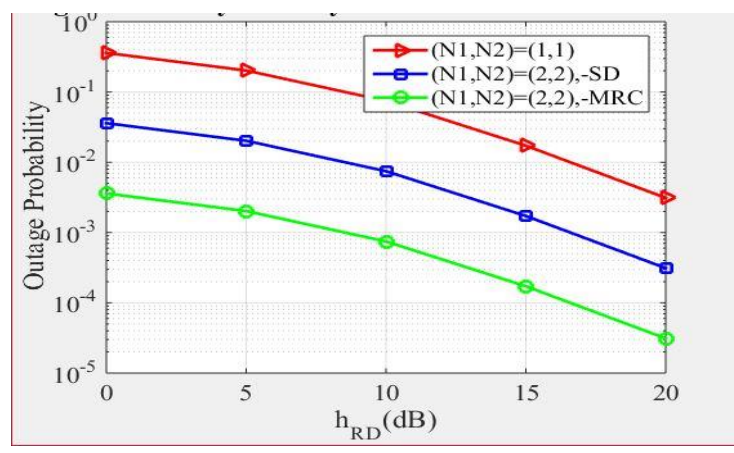

Fig.6. OP versus $h_{R D}$ for $P_{T}=50 m W, P_{D}=$ $50 \mathrm{~W}, h_{T R}=8$

It is shown that in the figure 6, OP decreases by increasing the number of antennas at the relay and destination. Number of antennas is increases received signal strength increases. So outage probability decreases. Compared to selection diversity technique MRC gives the better performance.

\section{CONCLUSION}

In this paper we evaluate the outage probability of the two-hop cooperative system with DF relays. Here we can achieve the Outage probability performance with the help of energy harvesting relays. Relays are charged by the RF radiation. Actually we can achieve this under the scenario OSRL Finally we conclude that

(1) Outage probability improved by the increasing energy harvesting time. If $\mathrm{EH}$ time is increases then there is chance to collect more DC voltage from RF signals.

(2) Data rate also show effect on the performance 


\section{REFERENCES}

[1] Gaofeng Pan, Yuanwei Liu, Hongjiang Lei," Performance analysis of cooperative communication with wireless energy harvesting relays over Nakagami-m fading channels, IEEE Trans.Commun, Aug 2017.

[2] L. Jiang, et al., "Social-aware energy harvesting device to device communications in $5 \mathrm{G}$ networks," IEEE Wireless Commun., vol. 23, no. 4, pp. 20-27, Aug. 2016.

[3]T.Le,K.Mayaram,andT.Fiez,"Efficientfarfieldradiofrequencyenergy harvesting for passively powered sensor networks," IEEE J. Solid-State Circuits., vol. 43, no. 5, pp. 12871302, May 2008.

[4] P. Grover and A. Sahai, "Shannon meets Tesla: Wireless information and power transfer," in Proc. IEEE Int'l Symp. Inf. Theory., pp. 23632367, June 2010.

[5] G. Pan, C. Tang, T. Li, and Y. Chen, "Secrecy performance analysis for SIMO simultaneous wireless information and power transfer systems," IEEE Trans. Commun., vol. 63, no. 9, pp. 3423-3433, Sept. 2015.

[6] G. Pan, H. Lei, Y. Yuan and Z. Ding, "Performance analysis and optimization for SWIPT wireless sensor networks," IEEE Trans. Commun., vol. 65, no. 5, pp. 2291-2302, May 2017.

[7] A. A. Nasir, X. Zhou, S. Durrani, and R. A. Kennedy, "Relaying protocols for wireless energy harvesting and information processing," IEEE Trans. Wireless Commun., vol. 12, no. 7, pp. 3622-3636, July 2013.

[8] Y. Liu, S. A. Mousavifar, Y. Deng, C. Leung and M. Elkashlan, "Wireless energy harvesting in a cognitive relay network," IEEE Trans. Wireless Commun., vol. 15, no. 4, pp. 24982508, Apr. 2016.

[9] Y. Liu, Z. Ding, M. Elkashlan, and H. V. Poor, "Cooperative non orthogonal multiple access with simultaneous wireless information and power transfer," IEEE J. Sel. Areas Commun., vol. 34, no. 4, Apr. 2016.

[10] H. Yu, D. Wang, G. Pan; R. Shi, J. Zhang, Y. Chen, "On outage of WPC system with relay selection over Nakagami-m fading channels," to appear in IEEE Trans. Veh. Tech..

[11] D. Wang, H. Yu, H. Lei, T. Li, J. Zhang, G. Pan and Y. Chen, "Outage of relay SWIPT with GSC and finite storage in Nakagami-m fading," to appear in IET Commun..

[12] H. Chen, Y. Li, J. L. Rebelatto, B. F. Uch^oFilho and B. Vucetic, "Harvest-then-cooperate: Wireless-powered communications," IEEE Trans. Sig. Proc., vol. 63, no. 7, pp. 1700-1711, Apr. 2015.

[13] T. Li, P. Fan, and K. B. Letaief, "Outage probability of energy harvesting relay-aided cooperative networks over Rayleigh fading channel," IEEE Trans Veh. Tech., vol. 65, no. 2, pp. 972-978, Feb. 2016.

[14] I. Krikidis, S. Timotheou, and S. Sasaki, "RF energy transfer for cooperative networks: Data relaying or energy harvesting?," IEEE Commun. Lett., vol. 16, no. 11, pp. 1772-1775, Nov. 2012.

[15] Y. Zhou, G. Pan, T. Li, et al., "Secrecy outage performance for partial relay selection schemes in cooperative systems," IET Commun., vol. 9, no. 16, pp. 1980-1987, Nov. 2015.

[16] Z. Ding, H. V. Poor, "Cooperative energy harvesting networks with spatially random users," IEEE Sig. Proc. Lett., vol. 20, no. 12, pp. 12111214, 2013.

[17] I. Krikidis, "Relay selection in wireless powered cooperative networks with energy storage," IEEE J. Sel. Areas in Commun., vol. 33, no. 12, pp. 2596-2610, Sept. 2015. 